\title{
Hereditary Malignant Urinary System Neoplasm
}

National Cancer Institute

\section{Source}

National Cancer Institute. Hereditary Malignant Urinary System Neoplasm. NCI

Thesaurus. Code C155950.

A malignant urinary system neoplasm that has developed in relatives of patients with a history of malignant urinary system neoplasm. 